\title{
Breathing pattern and respiratory mechanics in patients with amyotrophic lateral sclerosis
}

\author{
M. Vitacca*, E. Clini*, D. Facchetti**, M. Pagani*, M. Poloni+, \\ R. Porta*, N. Ambrosino*
}

Breathing pattern and respiratory mechanics in patients with amyotrophic lateral sclerosis, M. Vitacca, E. Clini, D. Facchetti, M. Pagani, M. Poloni, R. Porta, N. Ambrosino. (C) ERS Journals Ltd 1997.

ABSTRACT: The aim of this study was to evaluate the time course of breathing pattern and respiratory mechanics in patients with amyotrophic lateral sclerosis (ALS).

A study was conducted on 25 out of 38 eligible ALS patients. Neurological status, arterial blood gases (ABGs), spirometry, breathing pattern (minute ventilation $\left(V^{\prime} \mathrm{E}\right)$, tidal volume $(V T)$, respiratory frequency $(f \mathrm{R})$, duty cycle (duration of inspiration/duration of total breathing cycle $(t \mathrm{t} / \mathrm{t}$ tot $))$, respiratory drive $(P 0.1))$, respira-

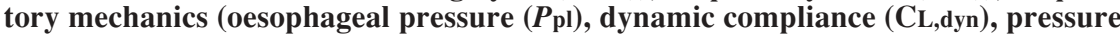
time product (PTP) and index (PTI), work of breathing (WOB)), and respiratory muscle $(\mathrm{RM})$ strength as assessed by maximal oesophageal pressure $(P \mathrm{pl}, \mathrm{max})$ were evaluated at presentation $(t 0)$ in all patients and after 6 months $(t 6)$ in 11 patients.

At $t 0$, the mean values of the degree of neurological impairment were $60 \pm 20$ and $103 \pm 30$ as assessed by the Norris scale and Medical Research Council (MRC) score, respectively. From the time of the first neurological symptom, survival time ranged 7-50 months. Diurnal ABGs were normal. A mild restrictive pattern was observed, a forced vital capacity (FVC) $<70 \%$ of predicted being present in $45 \%$ of patients, only FVC \% pred $(\mathrm{r}=0.59 ; \mathrm{p}<\mathbf{0 . 0 5})$, forced expiratory volume in one second (FEV1) $\%$ pred $(r=0.53 ; p<0.05)$ and survival $(r=0.64 ; p<0.05)$ showing a significant correlation with the Norris scale. A $P$ pl,max $<30 \mathrm{cmH}_{2} \mathrm{O}$ was associated with a significantly greater mortality, $P$ pl,max being correlated with survival $(r=0.79, p<0.05)$. At t6, $f \mathrm{R}, f \mathrm{R} / V_{\mathrm{T}}, P_{0.1} / P_{\mathrm{pl}}$ max, were significantly increased in comparison to to, while FVC $\%$ pred, vital capacity $(\mathrm{VC}) \%$ pred, FEV1 \% pred, $V$ T and $P$ pl,max were significantly reduced.

These results suggest a progressive deterioration in breathing pattern and in respiratory muscle strength with progression of disease. Eur Respir J 1997; 10: 1614-1621.
*Respiratory Division, and $* *$ Neurophysiology Laboratory, Salvatore Maugeri Foundation IRCCS, Medical Center of Gussago (BS), Italy. +3rd Neurologic Clinic, S. Paolo University Hospital, Milan, Italy.

Correspondence: M. Vitacca Fondazione Salvatore Maugeri IRCCS

Division di Pneumologia

Centro Medico di Gussago

Via Pinidolo 23

25064 Gussago (BS)

Italy

Keywords: Motor neuron disease neuromuscular disease respiratory function respiratory muscles

Received: September 201996 Accepted after revision February 191997
Amyotrophic lateral sclerosis (ALS) is a progressive, degenerative and fatal disease affecting both upper and lower motor neurons. The involvement of the respiratory muscles (RMs) leading to dyspnoea and respiratory failure, is the most common cause of death in these patients [1, 2]. Respiratory symptoms usually appear late, and the monitoring of lung and RM function have been reported to be the best prognostic indicators in these patients [3-5]. Most patients with ALS have reduced forced vital capacity (FVC) which is reported to be related to the presence of respiratory symptoms, and declines progressively with time $[3,4,6]$. RM impairment has been showed to be related to the stage of disease at presentation and to decline linearly with a great deal of interpatient variability [4].

Although other investigators have studied pulmonary mechanics in patients with neuromuscular diseases [7], to our knowledge there is little information on breathing pattern and respiratory mechanics [8], and on their time course in the natural history of the disease.

The objectives of this study were to describe the time course of breathing pattern and chest wall mechanical indices in patients with ALS and to determine the relationships, if any, between these indices and the progression of disease as assessed by common clinical scoring.

\section{Methods}

\section{Patients}

From October 1992 to February 1995, 38 consecutive patients examined at the neurology laboratory, with a diagnosis of ALS, either spinal or bulbar [9], were considered for the study. All patients underwent a complete standard laboratory radiograph, electromyography (EMG), multimodal evoked potentials and magnetic resonance imaging (MRI) of the brain and cervical spinal cord to exclude other diseases mimicking ALS. Diagnosis of definite ALS was made when the following criteria were met [10]: 1) muscle weakness and atrophy in at least two noncontiguous muscle groups, lasting 6 months or longer; 2) pyramidal tract involvement; and 3) EMG evidence of diffuse impairment of the anterior horn cells. Moreover, diagnosis of idiopathic ALS was 
accepted if exposure to infections and toxins or the presence of neoplasm, metabolic disorders or other progressive diseases were ruled out. No patient suffered from familial ALS. Exclusion criteria were: 1) presence of an episode of acute respiratory failure (ARF) at the time of presentation requiring mechanical ventilation (MV); 2) presence of a tracheotomy; and 3) inability to perform respiratory measurements.

Most patients were receiving conventional symptomatic therapy (nonsteroid anti-inflammatory drugs, amitriptyline). None of the patients underwent steroid or immunosuppressive treatment, or received other drugs known to affect RM function [11]. Informed consent was obtained verbally. The study was approved by the Ethics Committee of the Salvatore Maugeri Foundation IRCCS.

\section{Measurements}

At baseline (t0) and at 6 months ( $t 6)$, patients were submitted to the following monitoring.

Anthropometric data. Age, sex, height and weight were recorded. Percentage of ideal body weight (\%IBW) was computed referring to the Metropolitan Life Insurance Company Table [12]. Body mass index (BMI) was calculated as body weight/height ${ }^{2}$.

Neurological status. Clinical neurological examination with serial motor function and disability evaluation was performed by the same two neurologists (DF and M. Poloni) with specific experience both of application of the scales and of clinical trials on ALS.

Rating scales. Fifteen muscles were examined manually and graded according to the British Medical Research Council (MRC) score. This score assesses the segmental force for upper and lower limbs, and decreases with worsening of force, the maximum value being 150: 80 for upper limbs and 70 for lower limbs [13]. Functional quantitative evaluation was performed by means of the Norris ALS scale. This disability score was developed specifically for ALS, and includes evaluation of the function of upper and lower limbs, also taking into account bulbar function. This score uses 34 items rated with a value from 0 to 3; and the normal score is 100 [14].

Survival. Survival time was considered as the total number of months from the first symptom to death.

Lung function tests. Dynamic lung volumes and flowvolume loops were assessed by means of a portable spirometer (Pony class 1 Type B; COSMED, Rome, Italy); both digital readout and paper tracings were obtained. The highest values of vital capacity (VC), FVC and forced expiratory volume in one second (FEV1) observed in three tests were considered and expressed as percentage of the predicted values ( $\%$ pred), according to European Respiratory Society (ERS) standards [15].

Daytime and night-time blood gases. Arterial blood gases (ABGs) were assessed by means of an analyser (EGA system; Ciba Corning, Rome, Italy), using blood samples drawn from the radial artery while the patients were breathing room air. Arterial Oxygen saturation $\left(\mathrm{Sa}, \mathrm{O}_{2}\right)$ was continuously monitored during the night in 19 out of 25 patients by means of a portable pulsed oximeter with an extensive solid-state memory (Pulsox 5; Minolta Camera, Osaka, Japan). The pertinent definition of desaturation was: percentage of recorded time spent with $\mathrm{Sa}_{\mathrm{a}} \mathrm{O}_{2}<90 \%$.

Breathing pattern and mechanics. In the patients and in 10 healthy control subjects, all data were recorded in the same session in a semi-recumbent position, shortly after admission. Flow was measured by means of a flow sensor (Var Flex Flow transducer; CP-100 Bicore, Irvine, CA, USA) connected to a mouthpiece. Airway pressure was measured through a catheter attached to the flow sensor. Pleural pressure $(P \mathrm{pl})$, estimated by measuring oesophageal pressure swings, were measured by means of an oesophageal catheter with a $10 \mathrm{~cm}$ balloon at the distal end (part No. 700-3-100; Bicore, Irvine, CA, USA) passed transnasally and positioned in the lower third of the oesophagus. The position of the catheter was checked by means of the occlusion technique [16]. The oesophageal balloon and the flow sensor were connected to a portable monitor (CP-100 Bicore) providing real time display of flow, volume, and $P \mathrm{pl}$ tracings. After $15 \mathrm{~min}$ of breathing with the oesophageal catheter and having reached a good level of confidence with the set, airway pressure, airway flow and $P \mathrm{pl}$ were transmitted to the monitor and recorded for $180 \mathrm{~s}$ after the patients had developed at least a $2 \mathrm{~min}$ "steady state" period in their breathing pattern. The final data were collected as the average of at least three breaths, eliminating those affected by artifactual $P$ pl variations. Minute ventilation ( $\left.V^{\prime} \mathrm{E}\right)$ and breathing pattern (tidal volume $(V \mathrm{~T})$, respiratory frequency $(f \mathrm{R}), f \mathrm{R} / V \mathrm{~T}$ ratio, respiratory timing and duty cycle (duration of inspiration/duration of total breathing cycle $(t \mathrm{I} / t$ tot $))$, mean inspiratory flow $(V \mathrm{~T} / \mathrm{tI})$ ) were assessed by the flow signal. $P$ pl swings were analysed, measuring peak amplitude from the immediately preceding end expiratory value. Patient work of breathing (WOB) was assessed by calculating the area under the $P \mathrm{pl}$ versus lung volume curve during the negative deflection of $P$ pl tracings [17]. Respiratory drive $\left(P_{0.1}\right)$ was calculated as the change in $P$ pl occurring between the time $100 \mathrm{~ms}$ prior to the start of airflow, and the onset of flow. From $P_{0.1}$ and $V \mathrm{~T} / t \mathrm{I}$, respiratory impedance $(P 0.1 / V \mathrm{~T} / t \mathrm{I})$ was calculated. Maximal static inspiratory $P \mathrm{pl}(P \mathrm{pl}, \max )$ was assessed by means of a Muller manoeuvre during a maximal inspiratory effort generated after manual occlusion of the flow transducer starting from functional residual capacity (FRC). The subjects were verbally encouraged to achieve maximal strength. The highest value (most negative $P \mathrm{pl})$ of three tests was considered in data analysis [18]. Pressure time index (PTI) was calculated as $P \mathrm{pl} /$ $P \mathrm{pl}$,max $\times t \mathrm{I} / t$ tot. Dynamic compliance (CL,dyn) and pressure time product (PTP), were also calculated from $P$ pl, flow and volume data. Reliability and accuracy of the Bicore CP-100 pulmonary device in spontaneously breathing patients has been demonstrated previously [19].

\section{Statistical analysis}

All the data (parametric and nonparametric) are expressed as mean $\pm \mathrm{SD}$. The normal distribution of all 
Table 1. - Demographic, anthropometric and neurological data of patients at presentation (to)

\begin{tabular}{lc}
\hline Patients $\mathrm{n}$ & 25 \\
Gender males/females & $12 / 13$ \\
Age on admission yrs & $65 \pm 10$ \\
Weight $\mathrm{kg}$ & $63 \pm 16$ \\
Ideal body weight \% & $94 \pm 5$ \\
Height cm & $168 \pm 9$ \\
BMI (males) $\mathrm{kg} \cdot \mathrm{m}^{-2}$ & $20 \pm 5$ \\
BMI (females) $\mathrm{kg} \cdot \mathrm{m}^{-2}$ & $16 \pm 6$ \\
Age at diagnosis yrs & $63 \pm 10$ \\
Duration of disease months & $25 \pm 29$ (range 6-37) \\
MRC score & $103 \pm 30$ \\
Norris scale & $60 \pm 20$
\end{tabular}

Values are presented as absolute number or mean \pm SD. BMI: body mass index; MRC: Medical Research Council.

baseline variables recorded was evaluated with Kurtosis, Skeweenes and Shapiro-Wilk's W Test. A two-sample t-test or the Mann-Whitney rank sum test was used for the nonparametric variables between: 1) patients dead and alive for all the baseline variables; and 2) the two different subgroups according to Norris severity. Spearman rank correlation was used to test and to indicate the relationships between the duration of disease, and functional and neurological variables. The survival analysis was estimated with the Peto-Prentice (General Wilcoxon) test. In order to use the parametric discriminant analysis and correlation tests, variables found to be nonparametric were transformed as proposed by ARMITAGE and BERRY [20]. Logistic stepwise regression analysis was performed among all previously described anthropometric, spirometric, blood gas, breathing pattern and mechanical variables, to confirm the data obtained with stepwise discriminant analysis. A p-value of less than 0.05 was considered significant. All the results of multiple comparison were corrected using the Bonferroni test.

\section{Results}

Thirty eight consecutive patients with ALS were considered for the study. Five out of 38 patients were excluded due to an ARF episode requiring MV at the time of presentation, two patients refused, four were unable to perform evaluation, and two had a tracheotomy. Twenty five patients satisfied the acceptance criteria: five (20\%) had bulbar involvement and 20 (80\%) spinal prevalence. Demographic, anthropometric and neurological status as assessed by MRC and Norris scores, and duration of disease at $t 0$ for patients in the study are shown in table 1 . At $t 0$, nutritional status as assessed by IBW was in the normal range (table 1) and did not show any significant change at $t 6$.

At $t 610$ out of 25 patients $(40 \%)$ had died (two out of five $(40 \%)$ with bulbar involvement and eight out of $20(40 \%)$ with spinal involvement), and four out of 15 survivors were unable to attend the session. Differences at presentation among patients according to the Norris scale and according to whether patients had died or survived are shown in tables 2, 3, 4 and 5. Patients presenting with more severe neurological impairment according to the Norris scale were not different for spirometric and blood gas values, but showed lower $V \mathrm{~T} /$ $t \mathrm{I}, V \mathrm{~T}, P$ pl,max and higher $f \mathrm{R} / V \mathrm{~T}, P_{0.1} / P_{\mathrm{pl}}$,max and PTI.
Table 2. - MRC scores and respiratory function at presentation according to the severity of neurological status

\begin{tabular}{lccc}
\hline & \multicolumn{2}{c}{ Norris scale } & \multirow{2}{*}{ p-value } \\
\cline { 2 - 3 } & $<60(\mathrm{n}=16)$ & $>60(\mathrm{n}=9)$ & \\
\hline MRC score & $93 \pm 24$ & $123 \pm 131$ & $<0.01$ \\
$\mathrm{VC} \%$ pred & $62 \pm 20$ & $78 \pm 18$ & $\mathrm{NS}$ \\
$\mathrm{FEV} 1 \%$ pred & $66 \pm 30$ & $71 \pm 31$ & $\mathrm{NS}$ \\
$\mathrm{FVC} \%$ pred & $64 \pm 24$ & $85 \pm 24$ & NS \\
$\mathrm{FEV}_{1} / \mathrm{VC} \%$ & $80 \pm 18$ & $77 \pm 17$ & $\mathrm{NS}$ \\
$\mathrm{Pa}_{\mathrm{a}, \mathrm{CO}_{2}} \mathrm{kPa}$ & $5.71 \pm 0.66$ & $5.18 \pm 0.66$ & $\mathrm{NS}$ \\
$\mathrm{Pa}_{\mathrm{a}} \mathrm{O}_{2} \mathrm{kPa}$ & $10.10 \pm 1.46$ & $11.43 \pm 1.19$ & $\mathrm{NS}$ \\
\hline
\end{tabular}

Values are presented as mean \pm SD. MRC: Medical Research Council; VC: vital capacity; FEV1: forced expiratory volume in one second; FVC: forced vital capacity; $\mathrm{Pa}_{\mathrm{a}} \mathrm{CO}_{2}$ : arterial carbon dioxide tension; $\mathrm{Pa}_{\mathrm{a}, \mathrm{O}_{2}}$ : arterial oxygen tension; $\%$ pred: percentage of predicted value; Ns: nonsignificant.

Table 3. - MRC scores and respiratory function at presentation according to outcome

\begin{tabular}{lccc}
\hline & \multicolumn{2}{c}{ Outcome } & \multirow{2}{*}{ p-value } \\
\cline { 2 - 3 } & Died (n=10) & Survived $(\mathrm{n}=15)$ & \\
\hline Age yrs & $70 \pm 9$ & $59 \pm 8$ & $<0.01$ \\
Norris score & $54 \pm 19$ & $66 \pm 20$ & NS \\
MRC score & $97 \pm 35$ & $109 \pm 29$ & NS \\
Duration of disease & $23 \pm 13$ & $27 \pm 35$ & NS \\
$\quad$ months & & & \\
VC \% pred & $35 \pm 10$ & $81 \pm 27$ & $<0.001$ \\
FEV1 \% pred & $46 \pm 32$ & $77 \pm 25$ & $<0.001$ \\
FVC \% pred & $51 \pm 33$ & $80 \pm 20$ & $<0.001$ \\
$\mathrm{FEV}_{1} / \mathrm{VC} \%$ & $72 \pm 21$ & $81 \pm 16$ & NS \\
$\mathrm{Pa}_{\mathrm{a}, \mathrm{CO}} \mathrm{kPa}$ & $5.8 \pm 0.8$ & $5.3 \pm 0.5$ & NS \\
$\mathrm{Pa}_{\mathrm{a}, \mathrm{O}_{2}} \mathrm{kPa}$ & $9.7 \pm 1.7$ & $11.0 \pm 1.4$ & NS \\
\hline $\mathrm{Va}$ & & &
\end{tabular}

Values are presented as mean \pm SD. For definitions, see legend to table 2 .

Table 4. - Breathing pattern and mechanics at presentation according to the severity of neurological status

\begin{tabular}{|c|c|c|c|c|}
\hline & \multirow[b]{2}{*}{$\begin{array}{l}\text { Controls } \\
(\mathrm{n}=10)\end{array}$} & \multicolumn{2}{|c|}{ Norris score } & \multirow{2}{*}{ value } \\
\hline & & $\begin{array}{l}<60 \\
(n=16)\end{array}$ & $\begin{array}{l}>60 \\
(\mathrm{n}=9)\end{array}$ & \\
\hline $\begin{array}{l}f \mathrm{R} \\
\text { breaths } \min ^{-1}\end{array}$ & $14 \pm 6$ & $23 \pm 7$ & $21 \pm 8$ & NS \\
\hline$V \mathrm{~T} / t \mathrm{I} \quad \mathrm{mL} \cdot \mathrm{s}^{-1}$ & $452 \pm 130$ & $339 \pm 110$ & $438 \pm 195$ & $<0.03$ \\
\hline$t \mathrm{I} / t$ tot & $0.41 \pm 0.06$ & $0.40 \pm 0.05$ & $0.40 \pm 0.08$ & NS \\
\hline $\begin{array}{l}V^{\prime} \mathrm{E} \\
\mathrm{L} \cdot \mathrm{min}^{-1}\end{array}$ & $8.1 \pm 2.2$ & $6.8 \pm 2.5$ & $8.7 \pm 4.0$ & NS \\
\hline$V \mathrm{~T} \mathrm{~mL}$ & $722 \pm 147$ & $353 \pm 169$ & $500 \pm 216$ & $<0.03$ \\
\hline$f \mathrm{R} / V \mathrm{~T}$ & $29 \pm 27$ & $99 \pm 65$ & $43 \pm 23$ & $<0.03$ \\
\hline$P \mathrm{pl} \quad \mathrm{cmH}_{2} \mathrm{O}$ & $7 \pm 2.2$ & $7.2 \pm 3.1$ & $7.5 \pm 3.0$ & NS \\
\hline $\begin{array}{l}\mathrm{CL}, \mathrm{dyn} \\
\mathrm{mL} \cdot \mathrm{cmH}_{2} \mathrm{O}^{-1}\end{array}$ & $154 \pm 55$ & $80 \pm 53$ & $117 \pm 71$ & NS \\
\hline$P 0.1 \quad \mathrm{cmH}_{2} \mathrm{O}$ & $1.2 \pm 0.4$ & $2 \pm 1.2$ & $1.5 \pm 1.0$ & NS \\
\hline $\begin{array}{l}P \text { pl,max } \\
\mathrm{cmH}_{2} \mathrm{O}\end{array}$ & $90 \pm 20$ & $25 \pm 12$ & $50 \pm 25$ & $<0.02$ \\
\hline$P_{0.1} / P_{\mathrm{pl}, \max }$ & $0.01 \pm 0.02$ & $0.08 \pm 0.02$ & $0.03 \pm 0.01$ & $<0.03$ \\
\hline $\begin{array}{l}\text { PTP } \\
\mathrm{cmH}_{2} \mathrm{O} \cdot \mathrm{s} \cdot \mathrm{min}^{-1}\end{array}$ & $172 \pm 63$ & $139 \pm 50$ & $144 \pm 42$ & NS \\
\hline PTI & $0.05 \pm 0.03$ & $0.17 \pm 0.06$ & $0.05 \pm 0.03$ & $<0.02$ \\
\hline WOB $\mathrm{J} \cdot \mathrm{L}^{-1}$ & $0.70 \pm 0.22$ & $0.65 \pm 0.10$ & $0.64 \pm 0.11$ & NS \\
\hline
\end{tabular}

Values are presented as mean $\pm \mathrm{SD} . f \mathrm{R}$ : respiratory frequency; $V \mathrm{~T}$ : tidal volume; $t \mathrm{I}$ : duration of inspiration; $t$ tot: duration of total breathing cycle; $V^{\prime} \mathrm{E}$ : minute ventilation; $P$ pl: oesophageal pressure; CL,dyn: dynamic compliance; $P_{0.1}$ : respiratory drive; $P$ pl,max: maximal oesophageal pressure; PTP: pressure time product; PTI: pressure time index; WOB: work of breathing; NS: nonsignificant. p-values are for comparison of those with Norris score $<60$ and $>60$. 
Table 5. - Breathing pattern and mechanics at presentation according to the severity of neurological status

\begin{tabular}{|c|c|c|c|}
\hline & \multicolumn{2}{|c|}{ Outcome } & \multirow{2}{*}{$\mathrm{p}$-value } \\
\hline & $\begin{array}{c}\text { Died } \\
(n=10)\end{array}$ & $\begin{array}{c}\text { Survived } \\
(\mathrm{n}=15)\end{array}$ & \\
\hline$f \mathrm{R}$ breaths $\cdot \min ^{-1}$ & $28 \pm 8$ & $20 \pm 6$ & NS \\
\hline$V \mathrm{~T} / t \mathrm{I} \quad \mathrm{mL} \cdot \mathrm{s}^{-1}$ & $350 \pm 100$ & $400 \pm 150$ & NS \\
\hline$t \mathrm{I} /$ tot & $0.44 \pm 0.04$ & $0.39 \pm 0.07$ & NS \\
\hline$V^{\prime} \mathrm{E} \quad \mathrm{L} \cdot \mathrm{min}^{-1}$ & $7 \pm 2$ & $8 \pm 3$ & NS \\
\hline$V \mathrm{~T} \mathrm{~mL}$ & $305 \pm 75$ & $440 \pm 213$ & $<0.03$ \\
\hline$f \mathrm{R} / V_{\mathrm{T}}$ & $111 \pm 48$ & $60 \pm 55$ & $<0.05$ \\
\hline$P$ pl $\quad \mathrm{cmH}_{2} \mathrm{O}$ & $7.8 \pm 4$ & $7.1 \pm 3$ & NS \\
\hline $\mathrm{CL}$,dyn $\mathrm{mL} \cdot \mathrm{cmH}_{2} \mathrm{O}^{-1}$ & $58 \pm 24$ & $116 \pm 68$ & $<0.05$ \\
\hline$P 0.1 \quad \mathrm{cmH}_{2} \mathrm{O}$ & $1.8 \pm 1.0$ & $1.7 \pm 1.2$ & NS \\
\hline$P$ pl,max $\mathrm{cmH}_{2} \mathrm{O}$ & $21 \pm 9$ & $42 \pm 23$ & $<0.02$ \\
\hline$P_{0.1 / P \mathrm{pl}, \max }$ & $0.07 \pm 0.02$ & $0.04 \pm 0.01$ & NS \\
\hline PTP $\mathrm{cmH}_{2} \mathrm{O} \cdot \mathrm{s} \cdot \mathrm{min}^{-1}$ & $147 \pm 59$ & $139 \pm 46$ & NS \\
\hline PTI & $0.22 \pm 0.19$ & $0.06 \pm 0.03$ & $<0.02$ \\
\hline WOB $\mathrm{J} \cdot \mathrm{L}^{-1}$ & $0.79 \pm 0.11$ & $0.62 \pm 0.10$ & NS \\
\hline
\end{tabular}

Values are presented as mean \pm SD. For definitions, see legend to table 4 .

At presentation, mean age (table 3 ), $f \mathrm{R} / V \mathrm{~T}$, and PTI (table 5) were higher for patients who had died during the follow-up in comparison to survivors, while $\mathrm{VC} \%$ pred, FEV1 \% pred, FVC $\%$ pred (table 3 ), VT, CL,dyn, and $P \mathrm{pl}, \mathrm{max}$ were more severely compromised (table 5).

The causes of deaths were: acute respiratory failure due to pneumonia (30\%); aspiration $(10 \%)$; severe arrhythmia (20\%); acute myocardial infarction (10\%); and acute respiratory failure not associated with infectious aetiology $(30 \%)$. The mean time of survival was $38 \pm 37$ months (range 7-50); mortality rate during the observation time (October 1992 to February 1995) was $76 \%$ (19 out of 25).

\section{Spirometry}

Baseline lung function data are shown in table 2. Patients showed a mild restrictive pattern as assessed by a $\mathrm{VC}<70 \%$ pred in 10 out of $25(40 \%)$ patients. Thirty one per cent of patients showed a flow-volume loop consistent with upper airway dysfunction according to GARCIAPACHON et al. [21]. No significant difference was found in dynamic volumes and $\mathrm{ABG}$ between patients with different severity of neurological impairment as assessed by the Norris score (table 2). Only FVC \% pred $(\mathrm{r}=0.59 ; \mathrm{p}<0.05)$ and FEV1 \% pred $(\mathrm{r}=0.53 ; \mathrm{p}<0.05)$ showed significant correlation with the Norris scale. Tables 4 and 5 show the time course of dynamic volumes in the individual 11 patients assessed also at $t 6$. At this time, VC was reduced in all patients, the mean values being reduced to almost half.

\section{Breathing pattern and respiratory mechanics}

Tables 6 and 7 present the means and the individual values at $t 0$ and $t 6$ of the 11 patients who were able to attend at $t 6$. In comparison to our laboratory normal data (tables 4 and 5), patients showed a significantly lower $P$ pl,max and $V \mathrm{~T}$, while $P 0.1 / P \mathrm{pl}, \max , f \mathrm{R} / V \mathrm{~T}, P_{0.1} / V \mathrm{~T} / t \mathrm{I}$ and PTI were significantly greater. Patients with a more severe level of neurological involvement (as assessed by a Norris score $<60$ ) showed lower $V \mathrm{~T}, V \mathrm{~T} / t \mathrm{I}$ and $P$ pl,max, and greater $f \mathrm{R} / V \mathrm{~T}$ ratio and PTI in comparison with patients with a Norris score $>60$ (table 4). No significant relationship was found between the measured parameters and length of disease. In comparison to $t 0$, the 11 patients able to perform respiratory measurements at t6 showed $f \mathrm{R}, f \mathrm{R} / V \mathrm{~T}, P_{0.1} / P_{\mathrm{pl}, \mathrm{max}}$, significantly increased (table 7), while FVC \% pred, VC \% pred, FEV1 $\%$ pred (table 6 ), $V \mathrm{~T}$ and $P$ pl,max (table 7 ) were significantly reduced.

\section{Arterial blood gases}

As shown in tables 2 and 3 , at $t 0$ mean values of $A B G$ were in the normal range. Only one patient showed a $P \mathrm{a}, \mathrm{O}_{2}<8 \mathrm{kPa}$; eight (32\%) showed a $\mathrm{Pa}_{\mathrm{a}} \mathrm{O}_{2}<9 \mathrm{kPa}$. Only three $(12 \%)$ out of 25 patients showed a $\mathrm{Pa}_{\mathrm{a}} \mathrm{CO}_{2}>6 \mathrm{kPa}$. At $t 6$, mean values of $\mathrm{ABG}$ were still in the normal range: six (54\%) patients showing a $P \mathrm{a}, \mathrm{CO}_{2} \geq 6 \mathrm{kPa}$ and three (27\%) patients showing a $P \mathrm{a}, \mathrm{O}_{2} \leq 8 \mathrm{kPa}$ (table 6). All 19 patients in whom it was possible to perform noninvasive monitoring of $\mathrm{ABG}$ showed periods of desaturation. The average time of recording spent with $\mathrm{Sa}_{\mathrm{a}} \mathrm{O}_{2}$

Table 6. - Time course of neurological and respiratory function $(n=11)$

\begin{tabular}{|c|c|c|c|c|c|c|c|c|c|c|c|c|c|c|c|c|}
\hline \multirow[b]{2}{*}{$\mathrm{Pt}$} & \multicolumn{2}{|c|}{ MRC score } & \multicolumn{2}{|c|}{ Norris scale } & \multicolumn{2}{|c|}{$\mathrm{VC} \%$ pred } & \multicolumn{2}{|c|}{ FVC $\%$ pred } & \multicolumn{2}{|c|}{$\mathrm{FEV}_{1} \%$ pred } & \multicolumn{2}{|c|}{$\mathrm{FEV}_{1} / \mathrm{VC} \%$} & \multicolumn{2}{|c|}{$P \mathrm{a}, \mathrm{CO}_{2} \mathrm{kPa}$} & \multicolumn{2}{|c|}{$P \mathrm{a}, \mathrm{O}_{2} \mathrm{kPa}$} \\
\hline & to & $t 6$ & $t 0$ & $t 6$ & to & $t 6$ & to & $t 6$ & to & $t 6$ & to & $t 6$ & $t 0$ & $t 6$ & to & $t 6$ \\
\hline 1 & 120 & 115 & 54 & 46 & 90 & 43 & 73 & 40 & 58 & 33 & 68 & 69 & 5.6 & 6.1 & 16.9 & 12.1 \\
\hline 2 & 90 & 80 & 40 & 32 & 25 & 20 & 20 & 15 & 16 & 10 & 92 & 90 & 6.1 & 6.1 & 9.8 & 13.0 \\
\hline 3 & 72 & 67 & 57 & 50 & 95 & 45 & 80 & 48 & 82 & 55 & 80 & 90 & 5.6 & 6.0 & 8.8 & 9.6 \\
\hline 4 & 122 & 93 & 58 & 29 & 87 & 24 & 78 & 40 & 91 & 43 & 99 & 90 & 6.0 & 6.8 & 8.6 & 9.3 \\
\hline 5 & 124 & 118 & 79 & 54 & 96 & 44 & 85 & 40 & 83 & 51 & 76 & 97 & 5.1 & 7.0 & 10.9 & 7.3 \\
\hline 6 & 85 & 76 & 50 & 52 & NA & NA & NA & NA & NA & NA & NA & NA & 4.7 & 5.1 & 11.8 & 11.8 \\
\hline 7 & 149 & 138 & 82 & 60 & 45 & 36 & 47 & 41 & 48 & 41 & 82 & 77 & 5.1 & 4.9 & 10.8 & 10.6 \\
\hline 8 & 106 & 70 & 66 & 51 & 24 & 15 & 35 & 20 & 32 & 15 & 92 & 90 & 5.3 & 5.7 & 6.9 & 7.3 \\
\hline 9 & 112 & 95 & 57 & 44 & 18 & 10 & 20 & 15 & 18 & 16 & 94 & 85 & 5.3 & 5.9 & 12.0 & 12.4 \\
\hline 10 & 83 & 44 & 54 & 38 & 77 & 39 & 56 & 37 & 62 & 35 & 93 & 77 & 5.1 & 4.4 & 12.0 & 10.4 \\
\hline 11 & 149 & 124 & 89 & 62 & 101 & 98 & 105 & 89 & 92 & 88 & 74 & 74 & 6.5 & 6.7 & 9.8 & 8.0 \\
\hline $\mathrm{Me}$ & 110 & 93 & 62 & 48 & 66 & 37 & 60 & 38 & 58 & 39 & 85 & 84 & 5.4 & 6.0 & 10.8 & 10.2 \\
\hline \multirow{2}{*}{$\begin{array}{l}\text { SD } \\
\text { p-valu }\end{array}$} & 26 & 29 & 15 & & 34 & 25 & \multirow{2}{*}{\multicolumn{2}{|c|}{$<0.001$}} & 29 & & \multirow{2}{*}{\multicolumn{2}{|c|}{10}} & \multirow{2}{*}{\multicolumn{2}{|c|}{0.0}} & \multirow{2}{*}{\multicolumn{2}{|c|}{2.0}} \\
\hline & \multicolumn{2}{|c|}{ e $<0.001$} & \multicolumn{2}{|c|}{$<0.0001$} & \multicolumn{2}{|c|}{$<0.04$} & & & \multicolumn{2}{|c|}{$<0.001$} & & & & & & \\
\hline
\end{tabular}

Individual and mean values at $t 0$ and $t 6$ and means at $t 6$ are those of the 11 subjects available at $t 6$. NA: not available; Pt: patient; t0: baseline value; t6: 6 months follow-up value; MRC: Medical Research Council. For further definitions, see legend to table 4 . 
Table 7. - Time course of breathing pattern and mechanics $(n=11)$

\begin{tabular}{|c|c|c|c|c|c|c|c|c|c|c|c|c|c|c|c|c|}
\hline \multirow[b]{2}{*}{$\mathrm{Pt}$} & \multicolumn{2}{|c|}{$\begin{array}{l}\quad f \mathrm{R} \\
\text { breaths } \cdot \mathrm{min}^{-1}\end{array}$} & \multicolumn{2}{|c|}{$\begin{array}{l}V \mathrm{~T} / t \mathrm{I} \\
\mathrm{mL} \cdot \mathrm{s}^{-1}\end{array}$} & \multicolumn{2}{|c|}{$t \mathrm{I} / t$ tot } & \multicolumn{2}{|c|}{$\begin{array}{c}V^{\prime} \mathrm{E} \\
\mathrm{L} \cdot \mathrm{min}^{-1}\end{array}$} & \multicolumn{2}{|c|}{$\begin{array}{l}V \mathrm{~T} \\
\mathrm{~mL} \\
\end{array}$} & \multicolumn{2}{|c|}{$f_{\mathrm{R}} / V_{\mathrm{T}}$} & \multicolumn{2}{|c|}{$\begin{array}{c}P_{\mathrm{pl}} \\
\mathrm{cmH}_{2} \mathrm{O}\end{array}$} & \multicolumn{2}{|c|}{$\begin{array}{c}C \mathrm{~L}, \text { dyn } \\
\mathrm{mL} \cdot \mathrm{cmH}_{2} \mathrm{O}^{-1} \\
\end{array}$} \\
\hline & to & $t 6$ & to & $t 6$ & to & $t 6$ & to & $t 6$ & $t 0$ & $t 6$ & $t 0$ & $t 6$ & $t 0$ & $t 6$ & $t 0$ & $t 6$ \\
\hline 1 & 10 & 16 & 214 & 214 & 0.39 & 0.39 & 5.0 & 6.7 & 500 & 420 & 20 & 38 & 9 & 10 & 150 & 143 \\
\hline 2 & 22 & 31 & 125 & 122 & 0.40 & 0.41 & 6.6 & 9.3 & 300 & 300 & 73 & 103 & 6 & 6 & 140 & 120 \\
\hline 3 & 17 & 17 & 151 & 147 & 0.42 & 0.43 & 6.5 & 4.4 & 380 & 260 & 45 & 65 & 6 & 7 & 130 & 100 \\
\hline 4 & 14 & 23 & 197 & 147 & 0.38 & 0.51 & 6.3 & 3.4 & 450 & 150 & 31 & 153 & 5 & 4 & 98 & 85 \\
\hline 5 & 25 & 38 & 163 & 157 & 0.51 & 0.53 & 12.5 & 7.6 & 500 & 200 & 50 & 190 & 5 & 6 & 44 & 40 \\
\hline 6 & 25 & 23 & 225 & 160 & 0.34 & 0.48 & 11.5 & 2.3 & 460 & 100 & 54 & 230 & 6 & 16 & 31 & 79 \\
\hline 7 & 16 & 15 & 145 & 142 & 0.46 & 0.47 & 6.4 & 5.4 & 400 & 360 & 40 & 42 & 13 & 8 & 75 & 39 \\
\hline 8 & 13 & 22 & 208 & 198 & 0.40 & 0.42 & 6.5 & 3.3 & 500 & 150 & 26 & 147 & 13 & 10 & 100 & 93 \\
\hline 9 & 26 & 26 & 130 & 124 & 0.41 & 0.43 & 8.3 & 9.1 & 320 & 350 & 81 & 74 & 3 & 2 & 105 & 87 \\
\hline 10 & 11 & 18 & 417 & 417 & 0.32 & 0.32 & 8.8 & 5.0 & 800 & 280 & 14 & 64 & 7 & 4 & 150 & 75 \\
\hline 11 & 15 & 16 & 183 & 179 & 0.41 & 0.42 & 6.8 & 8.0 & 450 & 500 & 33 & 32 & 6 & 5 & 120 & 130 \\
\hline Mean & 18 & 23 & 198 & 183 & 0.40 & 0.44 & 7.8 & 5.7 & 461 & 279 & 42 & 104 & 7.2 & 7.1 & 104 & 90 \\
\hline $\mathrm{SD}$ & \multirow{2}{*}{\multicolumn{2}{|c|}{$<0.01$}} & 81 & 83 & 0.05 & 0.06 & 2.4 & 2.4 & 133 & 124 & 21 & 67 & 3.2 & 3.9 & 40 & 33 \\
\hline p-value & & & \multicolumn{2}{|c|}{ NS } & \multicolumn{2}{|c|}{ NS } & \multicolumn{2}{|c|}{ NS } & \multicolumn{2}{|c|}{$<0.01$} & \multicolumn{2}{|c|}{$<0.01$} & \multicolumn{2}{|c|}{ NS } & \multicolumn{2}{|c|}{ NS } \\
\hline
\end{tabular}

Table 7. - (continued)

\begin{tabular}{|c|c|c|c|c|c|c|c|c|c|c|c|c|c|c|}
\hline \multirow[b]{2}{*}{$\mathrm{Pt}$} & \multicolumn{2}{|c|}{$\begin{array}{c}P_{0.1} \\
\mathrm{cmH}_{2} \mathrm{O} \\
\end{array}$} & \multicolumn{2}{|c|}{$\begin{array}{l}P \text { pl,max } \\
\mathrm{cmH}_{2} \mathrm{O}\end{array}$} & \multicolumn{2}{|c|}{$P_{0.1} / P_{\mathrm{pl}, \max }$} & \multicolumn{2}{|c|}{$\begin{array}{c}P_{0.1} / V \mathrm{~T} / \mathrm{tI} \\
\mathrm{cmH}_{2} \mathrm{O} \cdot \mathrm{L} \cdot \mathrm{s}^{-1} \\
\end{array}$} & \multicolumn{2}{|c|}{ PTP } & \multicolumn{2}{|c|}{ PTI } & \multicolumn{2}{|c|}{$\begin{array}{c}\text { WOB } \\
\mathrm{J} \cdot \mathrm{L}^{-1}\end{array}$} \\
\hline & to & $t 6$ & $t 0$ & $t 6$ & $t 0$ & $t 6$ & $t 0$ & $t 6$ & $t 0$ & $t 6$ & $t 0$ & $t 6$ & to & $t 6$ \\
\hline 1 & 2 & 2 & 44 & 27 & 0.05 & 0.07 & 9.3 & 9.3 & 152 & 140 & 0.10 & 0.14 & 0.65 & 0.91 \\
\hline 2 & 3 & 3 & 35 & 26 & 0.09 & 0.11 & 24.0 & 22.9 & 141 & 110 & 0.08 & 0.09 & 0.50 & 0.98 \\
\hline 3 & 2 & 3 & 32 & 10 & 0.06 & 0.30 & 13.2 & 20.0 & 150 & 140 & 0.07 & 0.30 & 0.66 & 0.87 \\
\hline 4 & 2.5 & 1 & 40 & 30 & 0.06 & 0.03 & 12.6 & 6.8 & 186 & 170 & 0.04 & 0.06 & 0.30 & 0.29 \\
\hline 5 & 1 & 3 & 31 & 20 & 0.03 & 0.15 & 3.0 & 3.2 & 105 & 250 & 0.08 & 0.14 & 0.20 & 0.34 \\
\hline 6 & 4 & 6 & 36 & 20 & 0.11 & 0.30 & 8.2 & 17.1 & 167 & 334 & 0.08 & 0.26 & 0.84 & 1.93 \\
\hline 7 & 4 & 1 & 40 & 33 & 0.10 & 0.03 & 27.5 & 7.0 & 231 & 149 & 0.14 & 0.11 & 1.19 & 0.56 \\
\hline 8 & 2.5 & 3 & 35 & 20 & 0.07 & 0.15 & 12.0 & 15.1 & 189 & 210 & 0.15 & 0.21 & 0.89 & 0.79 \\
\hline 9 & 1 & 1 & 45 & 10 & 0.02 & 0.10 & 15.3 & 16.1 & 200 & 250 & 0.03 & 0.08 & 0.99 & 0.98 \\
\hline 10 & 1 & 1 & 25 & 15 & 0.04 & 0.07 & 2.4 & 2.3 & 112 & 78 & 0.12 & 0.09 & 0.57 & 0.48 \\
\hline 11 & 1 & 1 & 36 & 25 & 0.03 & 0.04 & 5.5 & 5.5 & 174 & 112 & 0.10 & 0.10 & 0.61 & 0.44 \\
\hline Mean & 2.18 & 2.2 & 36.3 & 21.4 & 0.06 & 0.12 & 12.08 & 11.39 & 164.3 & 176.6 & 0.09 & 0.14 & 0.67 & 0.78 \\
\hline & 1.15 & 1.6 & 5.8 & 7.6 & 0.03 & 0.10 & 7.97 & 7.10 & 37.5 & 76.5 & 0.04 & 0.08 & 0.29 & 0.46 \\
\hline p-value & \multicolumn{2}{|c|}{ NS } & \multicolumn{2}{|c|}{$<0.0001$} & \multicolumn{2}{|c|}{$<0.04$} & \multicolumn{2}{|c|}{ NS } & \multicolumn{2}{|c|}{ NS } & \multicolumn{2}{|c|}{ NS } & \multicolumn{2}{|c|}{ NS } \\
\hline
\end{tabular}

Individual and mean values at $t 0$ and $t 6$ and means at $t 6$ are those of the 11 subjects available at $t 6$. For definitions, see legends to tables 4 and 6.

a)

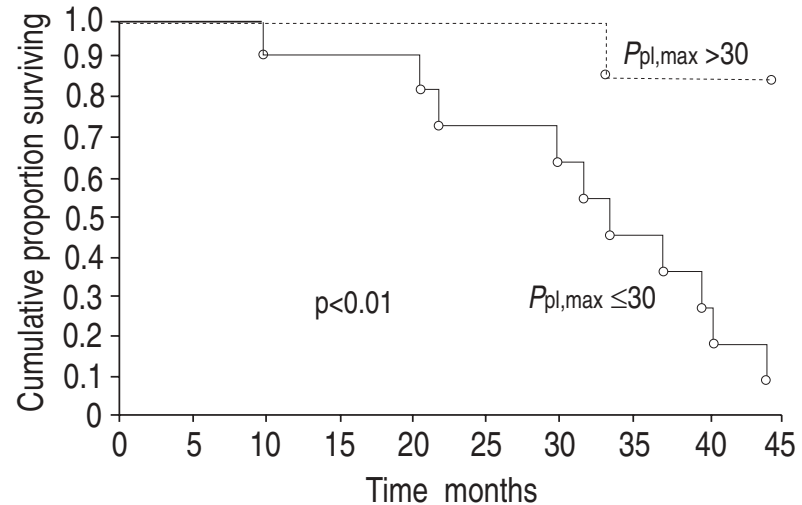

b)

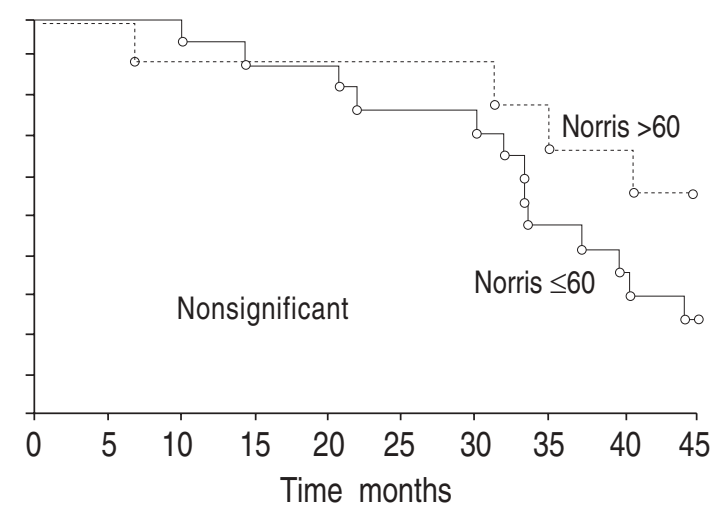

Fig. 1. - Cumulative survival rate according to: a) maximal oesophageal pressure $(P \mathrm{pl}, \mathrm{max})$ and $\mathrm{b})$ Norris score at presentation. $P$ pl,max $\mathrm{was}$ measured in $\mathrm{cmH}_{2} \mathrm{O}$. For further explantation see the text.

$<90 \%$ was $11 \pm 6 \%$ (range $3-82$ ); the mean nadir value was $75 \pm 10 \%$ (range $54-88$ ) and the mean number of desaturations was $3 \pm 2$ (range 1-10).

Figure 1 shows the survival curve of patients according to the baseline values of $P$ pl,max and Norris score. Patients with $P$ pl,max $<30 \mathrm{cmH}_{2} \mathrm{O}$ at presentation (16 out of 25 patients) showed a greater mortality in compari- son to the patients with $P$ pl,max $>30 \mathrm{cmH}_{2} \mathrm{O}$. During the same follow-up, 10 out of 25 patients who showed a Norris score $\leq 60$ at presentation showed a higher mortality in comparison to the patients with a Norris score $>60$. Spearman rank correlation analysis showed that $t 0 P \mathrm{pl}$,max correlated with survival (months before deaths) (rho= $0.79, \mathrm{p}<0.05)$. The same analysis showed that Norris 
scale correlated as well $(\mathrm{rho}=0.64 ; \mathrm{p}<0.05)$. A multivariate analysis excluding MRC and Norris scale showed that among all "respiratory", anthropometric and clinical history data, only age at the onset of disease predicted outcome (death vs survival) $(73.7 \%)$; the older the patient at onset of disease, the worse the prognosis.

\section{Discussion}

This study suffered from a high rate of dropouts. Of the 38 patients referred, only 25 satisfied the acceptance criteria. Of these, only 11 patients had a repeat study at 6 months. The others either died or were unable to perform forced manoeuvres. The results of this study suggest a progressive deterioration of the breathing pattern and of the RM strength with progression of the disease, as revealed by the clinical score. Although most of our results could be predicted as consequences of RM weakness, as in other chronic neuromuscular diseases, and other investigators have evaluated pulmonary mechanics in patients with ALS [7,8], the present study is more comprehensive and involves a larger number of patients than previous studies. ALS is a progressive degenerative disorder of the voluntary motor system that is characterized by loss and degeneration of motor neurons and their outflow tracts. The prognosis in ALS is extremely poor. Studies of 5 yr survival report rates ranging 18$42 \%$, with $50 \%$ of patients dying in the first 3 yrs [22]. Our patients showed a 45 month survival rate of $24 \%$ with a mean survival time of 38 months and a large degree of intersubject variability.

\section{Neurological impairment}

To evaluate neurological impairment, we used the Norris scale, which, in its original or modified form, is the most widely used scoring system in ALS [14]. This scale was specifically developed to determine an objective index of the progression of the disease in clinical trials and for the assessment of drug efficacy. It includes self-reported ability to perform some activities (chewing, swallowing, dyspnoea) and observed impairment in neurological functions (speech, standing, walking) or data from objective examination (muscular and tongue hypertrophy). Recently, a number of more articulate scales have been proposed for monitoring ALS progression [6, 23-25].

\section{Spirometry}

Our patients showed a mild restrictive pattern; therefore, they may be considered as suffering from an initial respiratory involvement. Abnormalities in pulmonary function, including reduced FVC declining progressively over time, are reported [3-5, 26]. NAKANO et al. [27] were among the first to report serial pulmonary function studies in ALS. They measured spirometry and diffusing capacity in 25 patients, 9 months after onset and at 15,22 and 33 months. Initial mean VC was within the normal range and averaged $58 \%$ pred at final measurement. Ringel et al. [6] showed that the decline in pulmonary function as assessed by a megascore proposed by ANDRES et al. [24] most closely correlated with death. Thus, spirometry is considered of value, not only in detecting early respiratory involvement in ALS, but also in predicting the course of respiratory failure $[5$, 6]. Thirty one per cent of patients showed abnormalities of the maximal flow-volume loop consistent with upper airway dysfunction [28]. This finding was reported to occur often, but not exclusively, in patients with bulbar manifestations and seems to be unrelated to prognosis [29].

\section{Arterial blood gases}

Some patients develop hypercapnia at a relatively early stage of the disease, but, in general, hypercapnia is reported to occur as a preterminal event [7]. Our finding of hypercapnia in only $13 \%$ of cases is in keeping with literature showing that gas exchange is well maintained until loss of lung volume becomes very severe ( $\mathrm{FVC}=20 \%$ pred) $[3,5]$. Breathing during sleep is commonly reported to be altered in many patients with other chronic neuromuscular disorders, even in patients such as ours who retain normal gas exchange during daytime; specifically, their pattern of breathing during sleep is fragmented with frequent episodes of hypopnoea and apnoea that are associated with hypercapnia and hypoxaemia [29]. Although formal sleep studies were beyond the aims of our study, noninvasive monitoring of night-time $\mathrm{Sa}_{\mathrm{a}} \mathrm{O}_{2}$ in our patients is in keeping with these data. Oxygen desaturation leads to disrupted sleep with frequent arousals, daytime headaches and somnolence, as found in our patients.

\section{Respiratory muscle function}

Weakness of the RM is an obligatory component of ALS. Moreover, progression in ALS is faster than in most other chronic neuromuscular disorders, explaining why respiratory failure is the most common cause of death [7]. In patients with chronic RM weakness, VC has been reported to be correlated with RM strength, but reductions in $\mathrm{VC}$ are considered to be greater than anticipated for the degree of RM weakness [7, 30]. In our study, RM strength, as assessed by $P$ pl,max, was reduced. BLACK and HyatT [31] found that ALS patients with near normal VC, like the patients in the present study, often had significant decreases in maximal inspiratory and expiratory pressures, the grade of dyspnoea correlating with the degree of RM impairment. Our results seem to indicate that in ALS patients, reduction in RM strength as assessed by $P$ pl,max may be observed at an early stage of respiratory involvement when lung volumes are still relatively preserved. On the other hand, although patients unable to perform respiratory manoeuvres were excluded by the study, RM strength as measured with a mouthpiece may be affected to a variable degree by the strength of facial muscles as opposed to that of the RM. This could explain, to some extent, the finding of a greater reduction of $P$ pl,max than $\mathrm{VC}$ in our patients and confirm the usefulness of assessing RM strength in an early phase of the disease. Alternatively, the partial discrepancy between the reduction of $P \mathrm{pl}$,max 
and VC could be also explained by a more reduced performance of the inspiratory muscle (IM) than of the expiratory muscles. In the absence of assessment of expiratory muscle function, this remains speculative. Furthermore, analysis showed that impairment of RM strength as assessed by $P$ pl,max was a predictive factor of survival.

\section{Breathing pattern and ventilatory drive}

Patients with a more severe level of neurological involvement showed significantly reduced values of $V \mathrm{~T}$ with increased $f \mathrm{R} / V \mathrm{~T}$ ratio. Patients with $\mathrm{RM}$ weakness breathe faster and with a smaller $V \mathrm{~T}$ than healthy subjects [32]. Interestingly, $\mathrm{Pa}, \mathrm{CO}_{2}$ changed only mildly over time despite a large reduction in $V^{\prime} \mathrm{E}$ (with a rapid shallow breathing observed at $t 6$ ), as in patients with a restrictive ventilatory defect of primary intrapulmonary origin [7]. $P 0.1$ and $V \mathrm{~T} / t \mathrm{I}$ ratio were not different from normal values. Measurement of $P 0.1$ and $V \mathrm{~T} / t \mathrm{I}$ ratio provide an estimate of central respiratory drive in such patients. It appears that the central respiratory drive is, in general, well preserved in patients with chronic neurological disorders $[7,32] . P 0.1$ is an index that reflects both the neural drive to, and the resulting force output of, the IM [33]. Although $P_{0.1}$ is used as an index of neural output to IM in normal subjects, in patients with reduction in IM strength, $P_{0.1}$ absolute values may underestimate the effective neural drive. $P 0.1 / P \mathrm{pl}$,max ratio was used as an index of central respiratory output normalized for IM strength [34] and was found to be increased in patients in this study in comparison to healthy control subjects. $P_{0.1} / V \mathrm{~T} / t \mathrm{I}$, an index of pulmonary impedance [35], was greater in patients than in controls and increased with time. Assessment of breathing pattern when using invasive devices, as well as using mouthpieces and noseclips, may have limitations. Nevertheless, our data show a reduction in $V \mathrm{~T}$, while artifacts associated with invasive measurements lead to higher $V \mathrm{~T}$ and $V^{\prime} \mathrm{E}$ [36].

\section{Respiratory mechanics}

In our patients with more severe neurological involvement, PTI, also referred to as the tension time index (TTI), was found to be in the threshold range of diaphragmatic fatigue mainly due to reduced $P$ pl,max. With the limitations of the few patients studied in the follow-up, PTI did not worsen with time. BELLEMARE and GRASSINO [37] showed that a TTI of 0.15-0.18 may be considered a critical level for development of diaphragmatic fatigue while the TTI for accessory IM was reported to be 0.30 or smaller [38]. TTI can be found to be increased by any combination of increased resistance, decreased compliance, IM weakness and malnutrition [39]. PTP was not different from healthy control subjects. PTP has been shown to be an index of energy consumption of the IM indicating the patient's effort to breathe [40].

In conclusion, patients with amyotrophic lateral sclerosis with mild restrictive ventilatory pattern may show reduction in respiratory muscle function, rapid shallow breathing and a preserved neural drive. A severe re- duction in inspiratory muscle strength is associated with a worse prognosis. The results of this study suggest a progressive deterioration of the breathing pattern and of the respiratory muscle strength with progression of the disease, as revealed by the clinical score.

\section{Appendix}

CL,dyn was calculated as follows:

$$
\mathrm{CL}, \mathrm{dyn}=V \mathrm{~T} /(P \operatorname{tp} 1-P \operatorname{tp} 2)
$$

where $V \mathrm{~T}=$ tidal volume; $P$ tp $1=$ transpulmonary pressure at maximum volume zero flow; $P$ tp $2=$ transpulmonary pressure at minimum volume zero flow.

PTP was calculated with the following formula:

$$
\left.\mathrm{PTP}=\int\left(P_{\text {ee }}-P_{\text {oes }}\right)+\left(\mathrm{Vol} / \mathrm{C}_{\mathrm{cw}}\right)\right) \mathrm{d} t / t
$$

where Pee = end-expiratory oesophageal pressure; Poes $=$ current oesophageal pressure; Vol $=$ current $V \mathrm{~T}$; $\mathrm{C}_{\mathrm{cw}}=$ chest wall compliance (estimated chest wall compliance equal to $\left.200 \mathrm{~mL} \cdot \mathrm{cmH}_{2} \mathrm{O}^{-1}\right) ; \mathrm{d} t=$ sample time; $t$ $=$ duration of breath in minutes.

\section{References}

1. Tandon R, Bradley WG. Amyotrophic lateral sclerosis, part 1: clinical features, pathology and ethical issues in management. Ann Neurol 1985; 18: 271-280.

2. Caroscio JT, Mulvihill MN, Sterling R, Abrams B. Amyotrophic lateral sclerosis: its natural history. $\mathrm{Neu}$ rol Clin 1987; 5: 1-8.

3. Fallat RJ, Jewitt B, Bass M, Kamm B, Norris FH. Spirometry in amyotrophic lateral sclerosis. Arch Neurol 1979; 36: 74-80.

4. Schiffman PL, Belsh JM. Pulmonary function at diagnosis of amyotrophic lateral sclerosis. Rate of deterioration. Chest 1993; 103: 508-513.

5. Kaplan LM, Hollander D. Respiratory dysfunction in amyotrophic lateral sclerosis. Clin Chest Med 1994; 15: 675-681.

6. Ringel SP, Murphy JR, Alderson MK, et al. The natural history of amyotrophic lateral sclerosis. Neurology 1993; 43: 1316-1322.

7. De Troyer A, Estenne M. The respiratory system in neuromuscular disorders. In: Macklem PT, Roussos C, eds. The Thorax. New York, Marcel Dekker, 1995; pp. 2177-2212.

8. Kreitzer SM, Saunders NA, Tyler HR, Ingram RH. Respiratory muscle function in amyotrophic lateral sclerosis. Am Rev Respir Dis 1978; 117: 437-447.

9. Munsat TL, Bradley WG. Amyotrophic lateral sclerosis. In: Tyler H, Dawson D, eds. Current Neurology. Boston, Houghton Miffin, 1979.

10. World Federation of Neurology Research Group on neuromuscular diseases. Subcommittee on motor neuron diseases "El Escorial World Federation of Neurology Criteria for the diagnosis of Amyotrophic Lateral Sclerosis" J Neurol Sci 1994; 124 (Suppl.): 96-107.

11. Aubier M, Decramer M. Respiratory muscle pharmacology. In: Macklem PT, Roussos C, eds. The Thorax. New York, Marcel Dekker, Part C, 1995; pp. 2341-2366.

12. Metropolitan Life Insurance Company. Statistical Bulletin 1977; 58: 5.

13. Medical Research Council. Aids to the investigation of 
peripheral nerve injury. War memorandum, 2nd Edn. London, HSMO, 1943; 11-46.

14. Norris FH, Calanchini PR, Fallat RJ, Pancharis S, Jewett B. Administration of guanidine in amyotrophic lateral sclerosis. Neurology 1974; 24: 721-728.

15. Quanjer Ph, Tammeling JE, Cotes JE, Pedersen OF, Peslin R, Yernault JC. Report working party standardization of lung function tests European Community for Steel and Coal: lung volumes and forced ventilatory flows. Eur Respir J 1993; 6: Suppl. 16, 5-40.

16. Baydur A, Behrakis K, Zin WA, Jaeger M, Milic-Emili J. A simple method for assessing the validity of the esophageal balloon technique. Am Rev Respir Dis 1982; 126: 788-791.

17. Otis AB. The work of breathing. In: Fenn WO, Rahn $\mathrm{H}$, eds. Handbook of Physiology. Washington DC, American Physiological Society, 1964; pp. 463-476.

18. Nava S, Ambrosino N, Crotti P, Fracchia C, Rampulla C. Recruitment of some respiratory muscles during three maximal inspiratory maneuvers. Thorax 1993; 48 : 702-707.

19. Vitacca M, Clini E, Porta R, Foglio K, Ambrosino N Acute exacerbation in patients with COPD requiring hospital admission: correlates of short-term outcome. Eur Respir J 1996; 9: 1487-1493.

20. Armitage P, Berry G. Statistical methods in medical research. Data editing. Oxford, Blackwell Scientific, 1988; pp. 358-370

21. Garcia-Pachon E, Marti J, Mayos M, Casan P, Sanchis J. Clinical significance of upper airway dysfunction in motor neuron disease. Thorax 1994; 49: 896-900.

22. McDonald ER, Wiedenfeld SA, Hillel A, Carpenter CL, Walter RA. Survival in amyotrophic lateral sclerosis. The role of psychological factors. Arch Neurol 1994; 51: 17-23.

23. Bensimon G, Lacomblez L, Meininger V, and the ALS/ Riluzole Study Group. A controlled trial of riluzole in amyotrophic lateral sclerosis. N Engl J Med 1994; 330: 585-589.

24. Andres PL, Finison LJ, Conlon T, Thibodeau LM, Munsat TL. Use of composite scores (megascores) to measure deficit in amyotrophic lateral sclerosis. Neurology 1988; 38: 405-408.

25. Hillel AD, Miller RN, Yorkston K, McDonald E, Norris FH, Konikow N. Amyotrophic lateral sclerosis severity scale. Neuroepidemiology 1989; 8: 142-150.
26. Braun SR. Respiratory system in amyotrophic lateral sclerosis. Neurol Clin 1987; 5: 9-31.

27. Nakano KK, Bass H, Tyler HR, Carmel RJ. Amyotrophic lateral sclerosis: a study of pulmonary function. Dis Nerv Sys 1976; 37: 32-35.

28. Owens GR, Murphy DMF. Spirometric diagnosis of upper airway obstruction. Arch Intern Med 1983; 143: 1331-1334.

29. Bye PTP, Ellis ER, Issa FG, Donnelly PM, Sullivan CE. Respiratory failure and sleep in neuromuscular disease. Thorax 1990; 45: 241-247.

30. Foglio K, Clini E, Facchetti D, et al. Respiratory muscle function and exercise capacity in multiple sclerosis. Eur Respir J 1994; 7: 23-28.

31. Black LF, Hyatt RE. Maximal static respiratory pressures in generalized neuromuscular disease. Am Rev Respir Dis 1971; 103: 641-649.

32. Baydur A. Respiratory muscle strength and control of ventilation in patients with neuromuscular disease. Chest 1991; 99: 330-338.

33. Whitelaw WA, Derenne JP, Milic-Emili J. Occlusion pressure as a measure of respiratory center output in conscious man. Respir Physiol 1975; 23: 181-199.

34. Gorini M, Ginanni R, Spinelli A, Duranti R, Andreotti L, Scano G. Inspiratory muscle strength and respiratory drive in patients with rheumatoid arthritis. Am Rev Respir Dis 1990; 142: 289-294.

35. Hussain SNA, Pardy RL, Dempsey JA. Mechanical impedance as determinant of inspiratory neural drive during exercise in humans. J Appl Physiol 1985; 59: 365-375.

36. Askanazi J, Silverberg PA, Foster RJ, Hyman AI, Milic Emili J, Kinney JM. Effects of respiratory apparatus on breathing pattern. J Appl Physiol 1980; 48: 577-580.

37. Bellemare F, Grassino A. Effect of pressure and timing of contraction on human diaphragm fatigue. J Appl Physiol 1982; 53: 1190-1195.

38. Zocchi L, Fitting JW, Majani U, Fracchia C, Rampulla C, Grassino A. Effect of pressure and timing of contraction on human rib cage muscles fatigue. Am Rev Respir Dis 1993; 147: 857-864.

39. Rochester DF, Esau SA. Malnutrition and respiratory system. Chest 1984; 85: 411-415.

40. Collett PW, Perry C, Engel LA. Pressure time product, flow and oxygen cost of resistive breathing in humans. J Appl Physiol 1985; 58: 1263-1272. 